\title{
O manejo com a trituração da vegetação favorece o incremento de matéria orgânica e as formas de fósforo no solo
}

\author{
The management with grinding of vegetation favors increase in organic matter and forms of \\ phosphorus in the soil. \\ J. C. de S. Júnior ${ }^{1}$, A. C. M. Braga ${ }^{2}$ C. R. C. dos Santos ${ }^{2 *}$, M. L. da S. Júnior ${ }^{2}$, G. \\ R. da Silva ${ }^{2}$, V. S. de $\mathrm{Melo}^{2}$ \\ ${ }^{I}$ Departamento de Ciência do Solo/Pós-graduação em Solos e Nutrição de Plantas, Escola Superior de Agricultura \\ Luís de Queiroz, 13418-900, Piracicaba-SP, Brasil \\ ${ }^{2}$ Instituto de Ciência Agrárias/Área de Ciência do Solo, Universidade Federal Rural da Amazônia, 66.077-830, \\ Belém-PA, Brasil
}

*rafaelsantos.18@hotmail.com

(Recebido em 20 de abril de 2015; aceito em 25 de junho de 2015)

\begin{abstract}
O objetivo do estudo foi avaliar os níveis de matéria orgânica e do fósforo com suas frações num argissolo amarelo sob cultivo de culturas anuais submetidos a pousios. A vegetação regenerativa nos períodos de pousio foi submetida aos manejos de corte, trituração e deposição sobre o solo ou queimada. Considerou-se, desta forma, três tratamentos: Sistema de corte e queima da floresta secundária (MQ); Sistema de corte e trituração da floresta secundária (MT); Floresta secundária em 40 anos de pousio (FS). Das amostras de solo de cada uma dessas três áreas, analisou-se: matéria orgânica (MO), fósforo total $(\mathrm{Pt})$, fósforo total lábil (Ptl), fósforo orgânico lábil (Pol), fósforo inorgânico lábil (Pil) e fósforo disponível (Pd). Os maiores valores de Pt foram encontrados na FS devido ao maior estado de equilíbrio edáfico encontrado neste tratamento. $\mathrm{O}$ solo sob manejo com deposição de resíduos vegetais (MT) apresentou os maiores valores de matéria orgânica e das frações total lábil (Ptl), orgânica lábil (Pol), inorgânica lábil (Pil) e disponível (Pd) em comparação com o manejo de queima da floresta (MQ) em regeneração, que, por sua vez, em comparação com o solo de uma floresta em pousio há quarenta anos apresentou menores valores dessas variáveis analisadas, exceto para Pt. O manejo com a conservação dos resíduos vegetais no sistema mostrou ser benéfico quanto ao incremento de matéria orgânica e aporte de frações de fósforo no solo, opostamente ao que apresentou o manejo com queima da vegetação em regeneração.
\end{abstract}

Palavras-chave: sustentabilidade do solo, agricultura itinerante, fracionamento do fósforo

This study aimed to evaluate the levels of organic matter and phosphorus with its fractions in a yellow argisol under annual crops growing submitted to fallow. The regenerative vegetation, in fallow periods was submitted to management with cut, grinding and deposition on soil or burning practices. Thus, three treatments were considered: System with Slash and burn of secondary forest (MQ); System with cut and grinding of secondary forest (MQ); Secondary forest in 40 years of fallow (FS). From samples of each area, it was analyzed: organic matter (MO); total phosphorus $(\mathrm{Pt})$; total labile phosphorus $(\mathrm{Ptl})$; organic labile phosphorus (Pol); inorganic labile phosphorus (Pil); and available phosphorus ( $\mathrm{Pd}$ ). The biggest values of $\mathrm{Pt}$ were found in FS due to the higher state of edaphic balance that was found in this treatment. The soil under management with deposition of plant residues (MT) showed the highest values of organic matter and of the total labile (Ptl), organic labile (Pol), inorganic labile (Pil) and available (Pd) fractions in comparison with forest burning management (MQ), which in turn, in comparison with the soil of a forest in fallow for 40 years, showed lowest values of this variables, except for Pt. The management with plant residues conservation in the system proved to be beneficial to increase organic matter and intake of phosphorus fractions in the soil, oppositely to presented by the forest burning system.

Keywords: soil sustainability, shifting cultivation, phosphorus fractionation 


\section{INTRODUÇÃO}

O fósforo $(\mathrm{P})$ é um dos elementos essenciais mais importantes para as plantas, sendo o mesmo responsável por diversas funções, como a composição de lipídeos, ésteres e ácidos nucléicos e a participação de reações fosforilativas através de compostos fosfatados com potencial energético (ATP e GTP)[13]. No solo, este nutriente apresenta-se sob várias frações, total, total lábil, orgânica e inorgânica lábil e disponível, as quais representam o seu grau de disponibilidade. Uma parte considerável destas frações de P é proveniente da matéria orgânica do solo (MO) [3].

Em solos de regiões tropicais, o fósforo é o nutriente mais limitante para as plantas cultivadas [16]. Nesses solos, o processo de fixação do $\mathrm{P}$ aos óxidos e hidróxidos de ferro e alumínio insolubiliza os fosfatos, diminuindo a disponibilidade deste nutriente para os vegetais [21]. A fixação do P também reduz a sua oferta para a população microbiana que, consequentemente, tem seu crescimento e diversidade reduzidos [7].

Nestas regiões, onde há a predominância de solos altamente intemperizados, o escoamento superficial aumenta a lixiviação de cátions básicos trocáveis e potencializa o caráter dreno das fontes de fósforo. Com a queima da vegetação para o plantio, as cinzas geradas também são lixiviadas pelo perfil de solo, tornando ainda mais efêmero o efeito de fertilização do solo oriundo da atividade de queima [5].

$\mathrm{Na}$ Amazônia, em geral, os sistemas tradicionais de produção agrícola utilizam a queima da floresta secundária como preparo de área [24]. A utilização do fogo promove a piromineralização da matéria orgânica $(\mathrm{MO})$ e disponibiliza os minerais imobilizados na biomassa para a solução do solo. Contudo, se usada consecutivamente, esta prática pode reduzir os compartimentos lábeis de $\mathrm{P}$ e aumentar as emissões de carbono para a atmosfera devido à oxidação da matéria orgânica do solo $[8,21]$.

Dentre as alternativas ao uso da queima têm-se a trituração, manual ou mecanizada, da floresta secundária em pousio. Na Região Amazônica, a EMBRAPA Amazônia Oriental, em parceria com pesquisadores da Alemanha, deu início ao Projeto SHIFT-Tipitamba no intuito de desenvolver tecnologias de aprimoramento da prática de trituração da vegetação como forma de preparo de área. Uma das principais criações do Projeto foi o implemento Tritucap, que tem como função cortar, triturar e depositar a biomassa em superfície [4].

De um modo geral, este sistema alternativo de manejo do solo apresenta como características o aumento considerável do teor de $\mathrm{MO}$ e consequente diminuição dos riscos de erosão e perda de bases por lixiviação, além da conservação da atividade microbiana. Além disto, tem-se a redução de acidentes com o uso do fogo e a lenta disponibilização de nutrientes com melhorias da fertilidade do solo à longo prazo $[10,21]$.

Uma das maneiras mais eficazes em avaliar os impactos dos preparos de área na disponibilidade do $\mathrm{P}$ tem sido estudar as modificações ocorridas nos compartimentos lábeis desse elemento como efeito do acúmulo ou redução da concentração de matéria orgânica do solo [25]. Segundo Zaia et al. (2008) [26] as proporções das frações lábeis de P em relação aos compartimentos deste nutriente variam conforme o preparo de área e cobertura do solo

Portanto, testou-se a hipótese que a capoeira triturada e depositada na superfície do solo, como forma de preparo de área para cultivos agrícolas e/ou florestais, em detrimento a sua queima, pode favorecer o incremento de matéria orgânica e dos compartimentos de $\mathrm{P}$, especialmente os de natureza lábil [4]. Em razão disso, objetivou-se avaliar o impacto da queima e da trituração da capoeira na concentração de matéria orgânica do solo, assim como nas formas total, total lábil, orgânica e inorgânica lábil e disponível de fósforo, disponível em Argissolo Amarelo da Amazônia oriental.

\section{MATERIAL E MÉTODOS}

\subsection{Caracterização da Área de Estudo}

O estudo foi conduzido no município de Igarapé-Açu, localizado na Microrregião Bragantina, nordeste do estado do Pará, no campus experimental Fazenda Escola de Igarapé-Açu (FEIGA) 
pertencente à Universidade Federal Rural da Amazônia ( $1^{\circ} 07^{\text {ee }} 15,5^{\prime \prime} \mathrm{S}$ e $47^{\circ} 36^{\text {ee }} 12,7^{\prime}$ ' W). O solo da área é classificado como Argissolo Amarelo Distrófico típico, textura média, segundo classificação proposta por Santos et al. (2013) [22], O clima da região é o megatérmico tropical úmido com pequena estação seca, classificado como Ami de acordo com sistema climatológico de Köppen, com temperatura e precipitação médias anuais de $27^{\circ} \mathrm{C}$ e de $2500 \mathrm{~mm}$.ano ${ }^{-1}$, respectivamente [4]. A vegetação predominante na região constitui-se de florestas secundárias com diferentes idades e estágios sucessionais. $\mathrm{Na}$ área, este tipo de vegetação é resultante, principalmente, da remoção de floresta primária com intuito de cultivos anuais ou perenes sendo que a ocupação por florestas ainda em estágio primário de desenvolvimento não chega a $10 \%$ [11].

\subsection{Delineamento Experimental e Amostragem}

As parcelas experimentais foram instaladas em janeiro de 2002, em área de floresta secundária onde não houve a prática de adubação por 40 anos. Para o presente estudo, adotou-se o Delineamento Inteiramente Casualizado (DIC) com quatro repetições. Os tratamentos foram: 1. Manejo com corte e queima da floresta secundária (MQ); 2 . Manejo com corte e trituração da floresta secundária (MT) e; 3. Floresta secundária com pousio de 40 anos como controle (FS).

Cada parcela experimental apresentou a dimensão de aproximadamente 2 ha. Após a instalação das parcelas aplicaram-se os tratamentos anualmente, sempre no início do período chuvoso da Região (janeiro a abril), com posterior plantio de culturas agrícolas, sem adubação (Tabela 1).

Tabela 1: Histórico de cultivo da área experimental preparada com corte-e-queima e corte-etrituração da vegetação da floresta secundária.

\begin{tabular}{lllc}
\hline Período & Cultura plantada & Pousio & $\begin{array}{c}\text { Tempo de pousio } \\
\text { - meses - }\end{array}$ \\
\hline $2002 / 2003$ & milho/mandioca & $2003 / 2004$ & 18 \\
$2005 / 2006$ & milho/mandioca & ---- & -- \\
$2006 / 2007$ & caupi/mandioca & $2008 / 2009$ & 24 \\
$2010 / 2011$ & milho/mandioca & $2012 / 2013$ & 24 \\
\hline
\end{tabular}

As amostras de solos foram coletadas em fevereiro de 2013 na camada 0-0,2 $\mathrm{m}$ em um transecto de $10 \times 40 \mathrm{~m}$, com auxílio de enxadeco e espátula reta. Cada amostra composta foi formada de três amostras simples, coletadas ao longo deste transecto.

\subsection{Análises Químicas}

As amostras compostas foram submetidas à secagem ao ar, sendo posteriormente peneiradas com peneira de malha de $2 \mathrm{~mm}$. Estas amostras foram destinadas ao Laboratório de Química do Solo/UFRA. Os solos peneirados com malha de $2 \mathrm{~mm}$ foram submetidas à avaliação da matéria orgânica (MO) e do fósforo disponível (Pd). Subamostras desse solo foram moídas em almofariz para passagem em malha de $0,1 \mathrm{~mm}$, com intuito de avaliar as seguintes frações de fósforo: fósforo total (Pt), fósforo total lábil (Ptl) e fósforo inorgânico lábil (Pil). Calculou-se o Pol a partir da subtração entre o Ptl e o Pil.

A matéria orgânica foi determinada por meio de sua oxidação com dicromato de potássio em meio ácido e o Pd foi extraído com solução duplo-ácida Mehlich-1, sendo ambas as análises realizadas de acordo com metodologia descrita por Silva (2009) [23]. A extração do Pt ocorreu em meio ácido [17] e as extrações de Ptl e Pil em meio alcalino, sendo estas últimas determinadas com base em Bowman \& Cole (1978) [2]. As formas de P foram quantificadas por espectrofotometria a $660 \eta \mathrm{m}$ utilizando como revelante o complexo molibdato-tartarato e ácido ascórbico (Murphy \& Riley, 1962) [15]. A concentração de MO foi determinada por meio da titulação com sulfato ferroso amoniacal [23]. 


\subsection{Análise Estatística}

Os dados foram submetidos à análise de variância (ANOVA) pelo teste $\mathrm{F}$ à $5 \%$ de significância $(p<0,05)$ e comparação de médias pelo teste de Duncan a $99 \%$ de probabilidade. Para o processamento estatístico utilizou-se o software R [19].

\section{RESULTADOS E DISCUSSÃO}

Os resultados obtidos nas análises de variâncias mostram que a concentração de MO foi maior no tratamento MT, apresentando diferença de 19,23\% quando comparada com o FS e de $34,61 \%$ quando comparado com MQ (Figura 1). Isso mostra que a deposição de resíduos vegetais oriundos da floresta favoreceu o incremento de MO no tratamento em que se utilizou a trituração da vegetação.

Perrin et al. (2014) [18] ao avaliarem o impacto do manejo de área com trituração e deposição de material oriundo da floresta secundária em superfície, em solo da Guiana Francesa, também verificaram incremento de matéria orgânica, associando o efeito à deposição de resíduos vegetais no solo. No estudo feito por Iwata et al. (2012) [9], foram analisados os efeitos de Sistemas Agroflorestais em comparação com uma área de corte e queima da vegetação e uma vegetação natural. A maior quantidade de MO nos SAFs com acúmulo de biomassa vegetal quando comparada com a agricultura de corte e queima converge com o resultado obtido no presente estudo.

Já para o Pt, a FS mostrou-se significativamente superior aos outros tratamentos, com diferenças de 16,54\% em comparação com MT e 70,02\% em comparação com MQ (Tabela 2). Em outras palavras, a redução da concentração de Pt no solo em resposta ao tratamento MQ foi mais acentuada que no tratamento MT, que representou em termos percentuais 70 e $16 \%$, respectivamente. Esses resultados sugerem que o tratamento MQ também influenciou a perda de outros compartimentos de $\mathrm{P}$, como a fração moderadamente lábil, moderadamente resistente $\mathrm{e}$ não lábil.

Tabela 2: Matéria Orgânica (MO) e Fósforo Total (Pt) em Argissolo Amarelo preparado com trituração e queimada vegetação

\begin{tabular}{ccc}
\hline Tratamentos & $\begin{array}{c}\text { MO } \\
\left(\mathbf{g ~ k g}^{-\mathbf{1}}\right)\end{array}$ & $\begin{array}{c}\mathbf{P t} \\
\left(\mathbf{m g ~ d m} \mathbf{~ d m}^{-\mathbf{3}}\right)\end{array}$ \\
\hline Floresta secundária (FS) & $20,50 \pm 1,22 \mathrm{~b}^{*}$ & $106,35 \pm 4,41 \mathrm{a}$ \\
Manejo com trituração (MT) & $26.18 \pm 2.91 \mathrm{a}$ & $88,87 \pm 5,10 \mathrm{~b}$ \\
Manejo com queima (MQ) & $18,44 \pm 2.37 \mathrm{c}$ & $32,37 \pm 2,59 \mathrm{c}$ \\
\hline Coeficiente de variação (\%) & 10,6 & 8,64
\end{tabular}

"Letras diferentes por coluna mostram variação significativa em nível de $1 \%$ pelo teste de Duncan.

Este resultado é esperado e se justifica pelo fato da FS estar em pousio por 40 anos, apresentando tal floresta um estágio de clímax, e estando, desta forma, em equilíbrio quanto à entrada e saída de nutrientes. Isto permite, portanto, a manutenção da concentração total de $\mathrm{P}$ elevada [3]. Comte et al. (2012) [4] verificaram na camada 0-0,05 m de Argissolo Amarelo sob floresta secundária concentração de Pt de $206 \mathrm{mg} \mathrm{kg}^{-1}$. Isto é explicado pelo fato de as camadas mais superiores do solo, naturalmente, apresentarem maiores níveis de $\mathrm{P}$ [16].

Os resultados das análises dos compartimentos lábeis de fósforo mostram que os valores de Ptl, Pol, Pil foram maiores no tratamento MT (Tabela 3). A diferença nas frações de P deste tratamento quando comparado com a FS foram, em termos percentuais, 33, 41 e 30\%, respectivamente. Isso mostra que a deposição de resíduos vegetais oriundos da floresta favoreceu o incremento de $\mathrm{MO}$ e esta, por sua vez, favoreceu formas lábeis de fósforo. 
Tabela 3: Frações lábeis de fósforo total lábil (Ptl), fósforo inorgânico lábil (Pil) e fósforo orgânico lábil (Pol) em Argissolo Amarelo preparado com trituração e queima da vegetação

\begin{tabular}{cccc}
\hline Tratamentos & Ptl & Pil & Pol \\
\cline { 3 - 4 } & & $\left(\mathbf{m g ~ d m}^{-3}\right)$ & \\
\hline Floresta secundária (FS) & $24,2 \pm 1,50 \mathrm{~b}^{*}$ & $6,3 \pm 1,38 \mathrm{~b}$ & $18,0 \pm 2,74 \mathrm{~b}$ \\
Manejo com trituração (MT) & $32,2 \pm 1,64 \mathrm{a}$ & $8,8 \pm 0,89 \mathrm{a}$ & $23,34 \pm 2,29 \mathrm{a}$ \\
Manejo com queima (MQ) & $12,6 \pm 0,86 \mathrm{c}$ & $4,3 \pm 0,55 \mathrm{c}$ & $8,3 \pm 1,74 \mathrm{c}$ \\
\hline Coeficiente de variação (\%) & 18,0 & 13,0 & 14,6 \\
\hline
\end{tabular}

*Letras diferentes por coluna mostram variação significativa em nível de $1 \%$ pelo teste de Duncan.

O tratamento MQ propiciou redução da concentração de Ptl, Pol e Pil em 93, 118 e 46\%, respectivamente, quando comparados à FS (Tabela 2). Esses resultados indicam que a queima afetou principalmente o compartimento Pol, confirmando o efeito negativo do fogo na fração orgânica de fósforo. Resende et al. (2011) [21] ao avaliarem o impacto da queima em Latossolo do cerrado verificaram que essa prática também reduziu o compartimento de Pol, o que pode ser associado à transformação da biomassa vegetal em cinzas através do fogo, com a rápida disponibilização de nutrientes por piromineralização. Tais cinzas são extremamente susceptíveis à lixiviação, sendo, portanto facilmente perdidas em condições de precipitação pluviométrica elevada, durante o período chuvoso [4].

Em outras palavras, sugere-se que a transformação da MO em cinzas, pela ação do fogo, e a alta pluviosidade da área de estudo $\left(2500 \mathrm{~mm}^{2} \mathrm{ano}^{-1}\right)$ facilitaram a lavagem deste material que contém o P, reduzindo a concentração da fração orgânica lábil nos solos com vegetação queimada [5]. Nesse sentido, a deposição superficial de resíduos triturados atua como oponente no processo de perda de solo, e consequentemente de nutrientes, além de prover a entrada de nutrientes no sistema por meio da ciclagem biogeoquímica, contribuindo com a reposição das extrações pelas raízes das plantas [20].

Quanto à concentração de $\mathrm{Pd}$, observou-se que não ocorreram diferenças significativas entre os tratamentos MQ e FS. Desta forma, a ausência de diferença estatística entre os dois tratamentos mostra que a queima da vegetação não alterou a concentração de Pd (Tabela 4). Em contrapartida, a trituração e deposição de resíduos sobre a superfície do solo aumentou a concentração desta fração, uma vez que se observa o CT significativamente maior do que os outros dois tratamentos quanto a esta variável.

Tabela 4: Fósforo Disponível (Pd) em Argissolo Amarelo preparado com trituração e queimada vegetação

\begin{tabular}{cc}
\hline Tratamentos & $\begin{array}{c}\text { Pd } \\
\left(\mathbf{m g ~ d m}^{-3}\right)\end{array}$ \\
\hline Floresta secundária (FS) & $2,55 \pm 0,40 \mathrm{~b}^{*}$ \\
Manejo com trituração (MT) & $4,60 \pm 0,19 \mathrm{a}$ \\
Manejo com queima (MQ) & $2,10 \pm 0,05 \mathrm{~b}$ \\
\hline Coeficiente de variação (\%) & 8,4 \\
\hline
\end{tabular}

"Letras diferentes por coluna mostram variação significativa em nível de $1 \%$ pelo teste de Duncan.

Lima et al. (2011) [12], ao avaliarem sistemas com deposição de fitomassa em comparação com floresta secundária e agricultura de corte e queima, obtiveram valores de fósforo disponível maiores nestes sistemas em que há acúmulo de material vegetal, em concordância com o presente estudo. Os autores também atribuíram tais resultados ao maior acúmulo de material orgânico devido à deposição de material neste sistema, com consequente aporte da fração disponível de $\mathrm{P}$.

Matos et al. (2012) [14] compararam o Pd de um solo sob Sistema Agroflorestal preparado com e sem queima da vegetação secundária e os resultados expuseram superioridade nos teores deste nutriente no solo, diferindo dos resultados encontrados no presente trabalho. 
Apesar do tratamento MT aumentar a concentração de Pd no solo em 2,05 mg dm ${ }^{-3}$, quando comparado ao tratamento FS, os resultados indicam que essa variável apresentou expressivo aumento em porcentagem, quando comparada às demais frações. Para Achat et al. (2010) [1], há uma relação direta do compartimento lábil com a disponibilidade do elemento, principalmente quando há mineralização de $\mathrm{P}$ orgânico em sistemas que adotam práticas com incremento de $\mathrm{MO}$, através da deposição de fitomassa pela trituração da vegetação.

Mesmo com o efeito benéfico que a capoeira triturada apresenta em aumentar à concentração das frações lábeis e, consequentemente disponibilizar P à solução do solo, a concentração de Pd foi considerada baixa quando comparada com as concentrações encontradas por Cravo et al. (2010) [6]. Isto ressalta a ideia de que os efeitos positivos da floresta triturada concentram-se, principalmente, no aumento da participação do compartimento lábil.

\section{CONCLUSÃO}

Comparativamente ao solo da floresta secundária com 40 anos de idade (FS) os solos das áreas sob cultivo de culturas anuais apresentaram teores divergentes de matéria orgânica, frações de fósforo e fósforo total. Considerando, portanto, os maiores valores ocorridos nas áreas com o manejo de corte, trituração e deposição da vegetação regenerada durante os períodos de pousio (MT) em relação à queima dessa vegetação (MQ), o primeiro demonstrou ser benéfico ao aporte de matéria orgânica e de formas de fósforo, opostamente ao que apresentou o manejo de queima da vegetação em regeneração. Desta maneira, recomenda-se a adoção do sistema com corte e trituração da biomassa vegetal como forma de preparo de área para o plantio.

\section{AGRADECIMENTOS}

Ao CNPq, pelo apoio logístico e financeiro ao desenvolvimento do trabalho.

\section{REFERÊNCIAS BIBLIOGRÁFICAS}

1. Achat DL, Backer MR, Zeller B, Pellerim S, Biename S, Morel C. Long-term organic phosphorus mineralization in Spodosols under forests and its relation to carbon and nitrogen mineralization. Soil Biology \& Biochemistry. 2010 Sep;42(5):1479-1490, doi:10.1016/j.soilbio.2010.05.020.

2. Bowman, R. A.; Cole, C. V. An exploratory method for fractionation of organic phosphorus from grassland soils. Soil Science. 1978 Feb;2(125):p.95-101, doi:10.1097/00010694-197802000-00006.

3. Brady NC, Neil RR. Elementos da Natureza e Propriedades dos Solos. Porto Alegre: Editora Bookman; 2013. 685 p.

4. Comte I, Davidson R, Lucotte M, Carvalho CJR, Oliveira FA, Silva BP, Rousseau GX. Physicochemical properties of soils in the Brazilian Amazon following fire-free land preparation and slash-and-burn practices. Agriculture, Ecosystems and Environment. 2012 Aug;1(15): 108-115, doi:10.1016/j.agee.2012.05.004.

5. Costa CFG, Figueiredo FO, Oliveira FA, Santos IPO. Escoamento superficial em Latossolo Amarelo distrófico típico sob diferentes agroecossistemas no nordeste paraense. Revista Brasileira de Engenharia Agrícola e Ambiental. 2013 Fev;2(17):162-169, doi:10.1590/S1415-43662013000200007.

6. Cravo, M. da S.; Viégas, I. de J. M.; Brasil, E. C. Recomendação de adubação e calagem para o Estado do Pará. Belém: Ed. Embrapa; 2010. 262p.

7. De Forest JL, Scott, LG. Available Organic Soil Phosphorus Has an Important Influence on Microbial Community Composition. Soil Biology \& Biochemistry. 2010 Dec;6(74):2059-2066, doi:10.2136/sssaj2009.0426.

8. Galang MA, Markewitz D, Morris LA. Soil phosphorus transformations under forest burning and laboratory heat treatments. Geoderma. 2010 Mar;155(4):401-408, doi:10.1016/j.geoderma.2009.12.026. 
9. Iwata BF, Leite LFC, Araújo ASF, Nunes LAPL, Gehring C, Campos LP. Sistemas agroflorestais e seus efeitos sobre os atributos químicos em Argissolo Vermelho-Amarelo do Cerrado piauiense. Revista Brasileira de Engenharia Agrícola e Ambiental. 2012 Abr;7(16):730-738, doi:10.1590/S141543662012000700005

10. Joslin AH; Markewitz D, Morris LA, Oliveira FA, Figueiredo RO, Kato OR. Five native tree species and manioc under slash and mulch agroforestry in the eastern Amazon of Brazil Plant growth and soil responses. Agroforestry Systems. 2011 Nov;1(81):1-14, doi:10.1007/s10457-010-9356-1.

11. Kato MSA., Kato OR, Denich M, Vlek PLG. Fire-free alternatives to slash-and-burn for shifting cultivation in the eastern Amazon region: the role of fertilizers. Field Crops Research. 1999 Jan;62:225-237.

12. Lima SS, Leite LFC, Oliveira FC, Costa DB. Atributos químicos e estoques de carbono e nitrogênio em argissolo vermelho-amarelo sob sistemas agroflorestais e agricultura de corte e queima no norte do Piauí. Revista Árvore. 2011 Fev; 1(35):156-168, doi:10.1590/S0100-67622011000100006.

13. Malavolta, E. Manual de Análises Químicas de Solos, Plantas e Fertilizantes. $1^{\mathrm{a}}$ Ed. Piracicaba: Editora Ceres, p. 163-193; 2006.

14. Matos FO, Castro RMS, Ruivo MLP, Moura QL. Teores de nutrientes do solo sob sistema agroflorestal manejado com e sem queima no Estado do Pará. Floram: Floresta e Ambiente. 2012 Set; 19(3):257-266, doi:10.4322/floram.2012.031.

15. Murphy J, Riley JPA. A modified single solution method for the determination of phosphorus in natural waters. Analytica Chimica Acta. 1962 Jan;27:31-36, doi:10.1016/S0003-2670(00)88444-5.

16. Novais RF, Alvares VH, Barros NF, Fontes RLF, Cantarutti RB, Neves JCL. Fertilidade do solo. $1^{\mathrm{a}}$ Ed. Viçosa (Brasil): Sociedade Brasileira de Ciência do Solo; 2007. Capítulo 8, Fósforo; p. 471-550.

17. Olsen, S. R.; Dean, L. A. Phosphorus. In: BLACK, C. A. Methods of soil analysis. Madison: American Society of Agronomy; 1965. 460, doi:10.2134/agronmonogr9.2.2ed.frontmatter.

18. Perrin AS, Fujisaki K, Petitjean C, Sarrazin M, Godet M, Garric B, Horth JC, Balbino LC, Filho AF, Machado PLOM, Brossard, M. Conversion of forest to agriculture in Amazon with the chop-andmulch method: does it improve the soil carbon stock? Agriculture, Ecosystem and Environment. 2014 Feb;184:101-114, doi:10.1016/j.agee.2013.11.009.

19. $\mathrm{R}$ Core Team. A language and environment for statistical computing. $\mathrm{R}$ foundation for statistical computing, 2013. Disponível em: http://www.r-project.org/.

20. Redel Y, Rubio R, Godoy R, Borie F. Phosphorus fractions and phosphatase activity in an Andisol under different forest ecosystems. Geoderma. 2008 Jun;3(145):216-221, doi:10.1016/j.geoderma.2008.03.007.

21. Resende JCF, Markewitz D, Klink CA, Bustamante MMC, Davidson EA. Phosphorus cycling in a small watershed in the Brazilian Cerrado: impacts of frequent burning. Biogeochemistry. 2011 Oct;3(105):105-118, doi: 10.1007/s10533-010-9531-5.

22. Santos HG, Jacomine PKT, Anjos LHC, Oliveira VA, Lumbreras JF, Coelho MR, Almeida JA, Cunha TJF, Oliveira JB. Sistema Brasileiro de Classificação de Solos. Brasília: Embrapa Solos; 2013. 353 p.

23. Silva FC. Manual de análises químicas de solos, plantas e fertilizantes. Brasília: Embrapa; 627p. 2009.

24. Trindade EFS, Kato OR, Carvalho EJM, Serafim ECS. Disponibilidade de fósforo em solos manejados com e sem queima no nordeste paraense. Amazônia: Ciência \& Desenvolvimento. 2011 Jun;12(6):7-9.

25. Turner, B. L.; Engelbrecht, B. M. J. Soil organic phosphorus in lowland tropical rain forests. Biogeochemistry. 2011;3(103):297-315, doi: 10.1007/s10533-010-9466-x.

26. Zaia CF, Gama-Rodrigues AC, Gama-Rodrigues EF, Machado RCR. Fósforo orgânico em solos sob agrossistemas de cacau. Revista Brasileira de Ciência do Solo. 2008 Out;5(32): 1987-1995, doi:10.1590/S0100-06832008000500020. 